BMJ Open

Sport \&

Exercise

Medicine

\section{High-intensity flywheel exercise and recovery of atrophy after 90 days bed-rest}

To cite: Belavý DL, Ohshima H, Rittweger J, et al. High-intensity flywheel exercise and recovery of atrophy after 90 days bed-rest. BMJ Open Sport Exerc Med 2017;3: 0000196. doi:10.1136/bmjsem-2016000196

Accepted 13 February 2017

\section{CrossMark}

${ }^{1}$ Centre of Muscle and Bone Research, Charité

Universitätsmedizin Berlin, Hindenburgdamm, Berlin, Germany

${ }^{2}$ School of Exercise and Nutrition Sciences, Institute for Physical Activity and Nutrition, Deakin University, Burwood, Victoria, Australia ${ }^{3}$ Department of Human Space Technology and Astronauts, Japan Aerospace Exploration Agency (JAXA), Tsukuba, Ibaraki, Japan ${ }^{4}$ German Aerospace Center, Institute of Aerospace Medicine, Cologne, Germany ${ }^{5}$ Department of Pediatrics and Adolescent Medicine, University of Cologne, Cologne, Germany

Correspondence to Dr Daniel L Belavý; belavy@ gmail.com

\section{ABSTRACT}

Aims To investigate differential muscle atrophy during bed-rest, the impact of a high-intensity concentriceccentric (flywheel) resistance exercise countermeasure and muscle recovery after bed-rest.

Methods Twenty-five healthy male subjects underwent 90 dayshead-down tilt bed-rest. Volume of individual lower-limb muscles was measured via MRI before, twice during and four times up to 1 year after bed-rest. Subjects were either inactive $(n=16)$ or performed flywheel exercise every third day of bed-rest $(n=9)$. Functional performance was assessed via countermovement jump.

Results On 'intent-to-treat' analysis, flywheel prevented atrophy in the vasti $(\mathrm{p}<0.001)$ and reduced atrophy in the hip adductor/extensor adductor magnus $(p=0.001)$ and ankle dorsiflexors/toe flexors (soleus $(p<0.001)$, gastrocnemius medialis $(p<0.001)$, gastrocnemius lateralis $(p=0.02)$, and tibialis posterior with flexor digitorum longus $(p=0.04))$. Flywheel exercise was not effective for the hamstrings, gracilis, sartorius, peroneals and anterior tibial muscles. Muscle atrophy in vasti, soleus, gastrocnemius medialis, gastrocnemius lateralis and adductor magnus correlated with losses in countermovement jump performance. Muscle volume recovered within 90 days after bed-rest, however long-term after bed-rest, the inactive subjects only showed significantly increased muscle volume versus prebed-rest in a number of muscles including soleus $(+4.3 \%)$, gastrocnemius medialis $(+3.9 \%)$ and semimembranosus $(+4.3 \%)$. This was not associated with greater countermovement jump performance.

Conclusion The exercise countermeasure was effective in preventing or reducing atrophy in the vasti, adductor magnus and ankle dorsiflexors/toe flexors but not the hamstrings, medial thigh muscles or peroneals and dorsiflexor muscles.

Trial registration number NCT00311571; results.

\section{INTRODUCTION}

Optimising countermeasures against musculoskeletal deterioration is a priority for space agencies around the world. With a long-term view to missions to Mars or a moon base, it is important to maintain the musculoskeletal system to enable completion of mission tasks in the hypogravitational fields on Mars or

\section{What are the new findings?}

In disuse, muscle atrophy occurs the fastest in the muscles of the lower-leg (especially soleus and gastrocnemius medialis) followed by those of the thigh (vasti, biceps femoris long head) and hip (quadratus femoris).

In ninety days of strict disuse, a fly-wheel exercise countermeasure reduced atrophy in the monoarticular knee, hip and ankle extensors, but did not impact the hamstrings or medial thigh muscles.

In the months after bed-rest, an overshoot of muscle volume recovery was observed in some muscles in the inactive control subjects with greater muscle size than before bed-rest.

moon, and also to safeguard the return to the Earth's $1 \mathrm{~g}$ environment. Prolonged bedrest is a methodology used to model the effects of spaceflight on the human body. ${ }^{1}$ Most of the musculoskeletal deterioration occurs in the lower quadrant of the body.

The literature to date ${ }^{2}$ points to the idea that muscle-specific higher-load resistance exercise appears to be more effective in maintaining muscle mass during prolonged bed-rest, with aerobic and/or low load type exercise being less effective. In microgravity, typical Earth-based implementations of resistance exercise are difficult. One approach to exercising in a gravity-independent manner is with the flywheel exercise device. ${ }^{3}$ During exercise with the flywheel device, resistance is determined by the effort applied to the device by the person during the concentric phase of movement. This energy is stored within the flywheels of the device and then returned as resistance during the eccentric phase. In this way, exercise is not dependent on force generation by, for example, a weight and pulley system. Flywheel exercise has been shown to be capable of generating greater eccentric muscle activation than with a standard gym weight system $^{4}$ and feasible for 
implementation in a space station simulation. ${ }^{5}$ In another model of unloading, unilateral lower-limb suspension, flywheel exercise of the knee extensors was shown to prevent knee extensor, but not plantarflexor, muscle atrophy ${ }^{6}$ and in an 84 days bed-rest study, it countered some of the metabolic changes in the vastus lateralis muscle. ${ }^{7}$ With the implementation of leg press and calf press type exercises on the flywheel device, our primary hypothesis was that flywheel exercise will reduce atrophy in hip, knee and calf extensor muscles in microgravity simulation (prolonged bed-rest).

Prolonged bed-rest is also a model of clinical disuse ${ }^{8}$ and can be informative clinically on which musculature is most affected and hence how to target rehabilitation programmes. While a series of works have examined muscle atrophy in the lower limb in disuse, ${ }^{9-17}$ yet less is known about atrophy of the deep muscles of the hip and the lower leg. A secondary aim of the current study was to examine which muscles of the lower limb atrophy the most. Furthermore, the recovery of the human body after strict disuse has not been well studied. This is important for estimating time windows of increased injury risk and designing rehabilitation programs. Our tertiary aim was to examine the time course of muscle atrophy after bed-rest and our tertiary hypothesis was that muscle atrophy during disuse will return to prebedrest levels in the months after bed-rest.

\section{METHODS}

\section{Study design, participants and study conduct}

Twenty-five healthy male subjects underwent 90 days of $6^{\circ}$ head-down tilt bed-rest (HDT) as part of the 'Long Term Bed Rest' (LTBR) Study at MEDES in Toulouse, France. The LTBR Study was supported by the European, French and Japanese space agencies (ESA, CNES and NASDA). The LTBR Study was approved by the Toulouse I ethics committee (CCPPRB Toulouse I) of the Rangueil University Hospital as well as the ethical committee of the Free University of Berlin. All subjects gave their informed written consent. Subjects were randomised to either a group performing high-intensity eccentric-concentric resistance exercise on the 'flywheel' exercise device (flywheel group; $\mathrm{n}=9$; $\operatorname{mean}(\mathrm{SD})$ age, height and weight: $31.0(5.5)$ years, $1.75(0.05) \mathrm{m}$ and $70.9(5.4) \mathrm{kg}$ ) or a group that performed no exercise (inactive group; $\mathrm{n}=16 ; 32.5$ (3.4) years, $1.74(0.04) \mathrm{m}$, $70.3(6.1) \mathrm{kg})$. Diet was strictly monitored and controlled during the in-house periods. Meals were prepared by the hospital kitchen to achieve energy intake with $30 \%$ fat, $15 \%$ protein and $55 \%$ carbohydrate and this was supervised by a dietician. Data on subjects' physical activity levels showed that 90 days after bed-rest, subjects' total physical activity scores were significantly less than before bed-rest. ${ }^{18}$

\section{Countermeasure exercise}

The flywheel exercise programme was designed to target muscle and bone in the lower limbs. The exercise device was designed to be gravity-independent. During the concentric contraction phase, the energy applied to the foot plate by the subject was transferred to the flywheels via a strap which was wound around the flywheels. Then, during the eccentric phase, the rotating flywheels provided resistance. Repetitions were performed as one continuous movement cycle, that is, movement was not ceased between each repetition. Prior to bed-rest all subjects performed two familiarisation sessions with the training apparatus on 2 days separated by $4-6$ days. In the first session, subjects practised the manoeuvres on the flywheel device at low to moderate effort intensities and in the second session, subjects were progressed to putting in maximum effort. During bed-rest, the flywheel group performed supine leg press exercises (4 sets of 7 repetitions, $2 \mathrm{~min}$ between sets; targeting the hip and knee extensor groups) with then $5 \mathrm{~min}$ rest and then calf-raise exercises (4 sets of 14 repetitions, 2 min between sets; targeting plantar flexors) every third day from the fifth day of bed-rest onwards. At the start of each set of exercises, two submaximal manoeuvres of the action (ie, leg press or calf-raise) were performed and these were immediately followed by the prescribed number of eccentric-concentric repetitions at maximum contraction. The subject was encouraged to put maximum effort into each concentric-eccentric loading cycle. After maximal concentric contraction at the initiation of the movement, the subject was instructed to start eccentric contraction (deceleration) once half of the range of motion was passed with then maximal effort into an eccentric contraction towards the end of the range of motion. Electrogoniometers positioned at the knee and ankle provided instantaneous feedback on joint position via a screen positioned in the subject's field of view.

\section{Magnetic resonance imaging}

A 1.0T Siemens Somatom Impact (Erlangen, Germany) scanner was used to conduct MRI before bed-rest (Baseline), on day 27 (HDT27) and day 89 (HDT89) of bed-rest, and then 13 days $(\mathrm{R}+13), 90$ days $(\mathrm{R}+90)$, 180 days $(R+180)$ and 360 days $(R+360)$ after bed-rest during the recovery phase. Both lower limbs, starting at the hip joint and extending to the feet, were scanned (see online supplementary figure 1). Images were then stored for offline analysis. To blind the operator (DLB) to group and study date, each data set was coded with a random number (obtained from www. random.org). The operator used ImageJ (http://rsb. info.nih.gov/ij/) to measure the area of muscles of the lower limb in every image on the right side of the body (see online supplementary figure 1). For the quadratus femoris and obturator internus muscles, based on our prior data ${ }^{19}$ it was deemed necessary to measure both 
left and right sides with the results averaged prior to further analysis.

\section{Countermovement jump}

For the comparison of changes in countermovement jump performance to muscle atrophy we used data from previously published work ${ }^{20}$ where detailed methodology of the countermovement jump test was reported. For the purpose of the correlation analyses, only data on percentage change between baseline and first testing session after bed-rest (day 3 after bed-rest; $\mathrm{n}=25$ ) and the last testing session in the recovery phase (180 days postbed-rest; $n=24)$ were used. We examined peak jump power; similar results are seen when assessing peak jump height or acceleration. The reproducibility of countermovement jump power in adult and elderly subjects is excellent ${ }^{21}{ }^{22}$ with data from our own laboratory ${ }^{23}$ on 24 men aged 20-45 years tested 4 days apart showing a between-day correlation of 0.93 for countermovement jump power. Percentage change in peak jump power versus before bed-rest was used in correlation analyses.

\section{Statistical analyses}

Analysis was done on an 'intent-to-treat' (ITT) approach. For the primary analysis, data were converted to fractional change in muscle volume and compared with the prebed-rest value. To examine whether the rates of atrophy differed between groups, non-linear mixed-effects modelling was used to calculate exponential decay rates per week of bed-rest $\left(e^{\mathrm{k} *}{ }^{\text {weeks }}\right.$; where $k$ is the decay rate coefficient and weeks is the week of bedrest). A factor of 'group', with subsequent analysis of variance (ANOVA), was examined to determine whether rate of muscle atrophy differed significantly between groups. To examine the recovery of the musculature after bed-rest, the data on whole muscle volume were used. A 'study-date' effect with a priori contrasts comparing each time point to baseline was evaluated. Linear mixed-effects models were implemented. For correlation analyses, Pearson's correlation coefficient was calculated between percentage change in muscle volume and percentage change in countermovement jump power. An $\alpha$ level of 0.05 was taken for statistical significance. To guard against false positives, all $\mathrm{p}$ values were adjusted by the false discovery rate method. The 'R' statistical environment (V.3.0.2, www.r-project.org) was used for all analyses.

\section{RESULTS}

One flywheel subject ceased the training protocol after 7 weeks bed-rest due to a previously unreported knee injury, but he completed the bed-rest study phase. This subject was treated as a flywheel subject in the ITT analysis. Data from one inactive subject were lost on HDT29. Two inactive subjects did not attend on $\mathrm{R}$ +90 and one of these also did not attend scanning on
$R+180$. One flywheel subject did not return for the final scanning session on $\mathrm{R}+360$.

\section{Effects in the inactive group}

In the inactive subjects, the soleus and gastrocnemius medialis showed the fastest rates of muscle atrophy (figure 1) both losing 28\% of their volume by end of bed-rest (table 1). This was followed by the remaining muscles of the lower leg (with the exception of the anterior tibial muscles), vasti, biceps femoris long head and semimembranosus (figure 1). The other members of the hamstrings, semitendinosus and biceps femoris short head, while atrophying, did not show as rapid atrophy of other members of the hamstrings. Of the muscles localised at the hip joint, quadratus femoris atrophied the fastest and of the medial thigh muscles, the greatest atrophy was seen in adductor magnus.

\section{Impact of flywheel exercise}

ANOVA showed that flywheel exercise significantly reduced the rate of atrophy of the posterior calf muscles soleus $(\mathrm{p}<0.001$; figure 1$)$, gastrocnemius medialis $(\mathrm{p}<0.001)$, gastrocnemius lateralis $(\mathrm{p}=0.02)$ and tibialis posterior with flexor digitorum longus $(p=0.04)$. At the thigh, flywheel exercise reduced atrophy of the vasti $(\mathrm{p}<0.001)$ and adductor magnus muscles $(p<0.001)$. Lower gluteus maximus and rectus femoris atrophied significantly in the inactive group and not in the flywheel group, but the differences between the groups were not statistically significant on ANOVA $(p>0.07)$. Similarly, gracilis atrophied significantly in the flywheel group but not in the inactive group, however the difference between groups in the rates of atrophy was not significant $(p=0.28)$. The flywheel exercise had no impact on atrophy of the hamstrings or other muscles of the thigh and hip.

\section{Long-term recovery of muscle volume after 90 days bed-rest} study

Of the muscles that atrophied during bed-rest, significant atrophy was still present 13 days after bed-rest (tables 1 and 2). By 90 days after 90 days bed-rest, all muscles returned to their prebed-rest volumes. In the inactive subjects only, some muscles showed hypertrophy in the months after bed-rest. Specifically, at the hip, adductor brevis was increased in volume on 180 days $(+6.0 \%, \mathrm{p}=0.008)$ and 360 days $(+4.9 \%, \mathrm{p}=0.040)$ of recovery and obturator externus also showed greater volume in recovery (90 days: $+8.6 \%, p=0.004 ; 180$ days: $+7.6 \%, p=0.007 ; 360$ days: $+6.4 \%, p=0.009)$. In the thigh, semimembranosus (180 days: $+2.7 \%$, $\mathrm{p}=0.019 ; 360$ days: $+4.3 \%, \mathrm{p}=0.020)$ and sartorius (180 days: $3.8 \%, \mathrm{p}=0.023 ; 360$ days: $+5.0 \%, \mathrm{p}=0.010$ ) were hypertrophied in the long term after bed-rest. In the calf, soleus $(180$ days: $+3.9 \%, \mathrm{p}<0.001 ; 360$ days: $+4.3 \%, \mathrm{p}=0.004)$ and medial gastrocnemius (360 days: $+3.9 \%, \mathrm{p}=0.040)$ also showed increased volume late in 
recovery. This effect was not seen in the flywheel group (table 1,2; figure 2).

\section{Relationship of muscle atrophy to peak lower limb performance}

At the end of bed-rest significant associations were seen between loss of muscle volume in the vasti, soleus, gastrocnemius medialis, gastrocnemius lateralis and adductor magnus and losses of countermovement jump performance (table 3). There were no positive correlations between muscle volume differences versus baseline and countermovement jump performance 180 days after bed-rest.

\section{DISCUSSION}

The current study permits some important insights into countermeasure exercise prescription. The flywheel exercise countermeasure was effective, compared with control, in preventing or reducing atrophy in the monoarticular knee extensors (vasti), mono articular hip extensors (adductor magnus) and ankle dorsiflexors/toe flexors. The actual exercise time was $7-9 \mathrm{~min}$ 'time under tension' with 20 min total training time including rest breaks. Importantly, these findings underscore ${ }^{19}$ that a short duration, high load, resistance exercise programme can be very effective in reducing muscle atrophy in bed-rest. Previous studies that have implemented low load, non-specific muscle exercises, were unsuccessful at reducing muscle atrophy: whole body vibration in standing in a squat position without exercise did not impact lower limb muscle atrophy, ${ }^{17}$ nor did whole body vibration implemented in supine position prevent spinal muscle atrophy. ${ }^{24}$ Furthermore, exercise protocols involving aerobic-based cycling ${ }^{25}$ and lower body negative pressure ${ }^{26}$ were largely ineffective at reducing lower limb muscle atrophy. An ergometerbased cycling exercise ${ }^{27}$ can be effective in reducing lower limb suspension induced muscle atrophy when higher intensities, and hence need for higher force muscle contraction, are implemented.

The countermeasure programme did not have an impact on atrophy in the hamstrings, which are important for ambulation. Of the hamstrings, semimembranosus and biceps femoris long head are primarily hip extensors $^{28}$ with semitendinosus playing more of a role in control of knee flexion. ${ }^{29}{ }^{30}$ In contrast, another research group $^{11}$ reported complete prevention of hamstring muscle atrophy due to their resistance exercise protocol in 20 days bed-rest when the leg press exercise was performed up to $110^{\circ}$ of hip flexion. This underscores the need for greater specificity of exercise prescription for the hamstrings.

Maintenance of the calf musculature in disuse bed-rest via exercise has, in a number of studies, proven difficult. In the current study the atrophy of the calf muscles was significantly reduced, but muscle atrophy still occurred: $20 \%$ of soleus muscle volume was lost in the flywheel

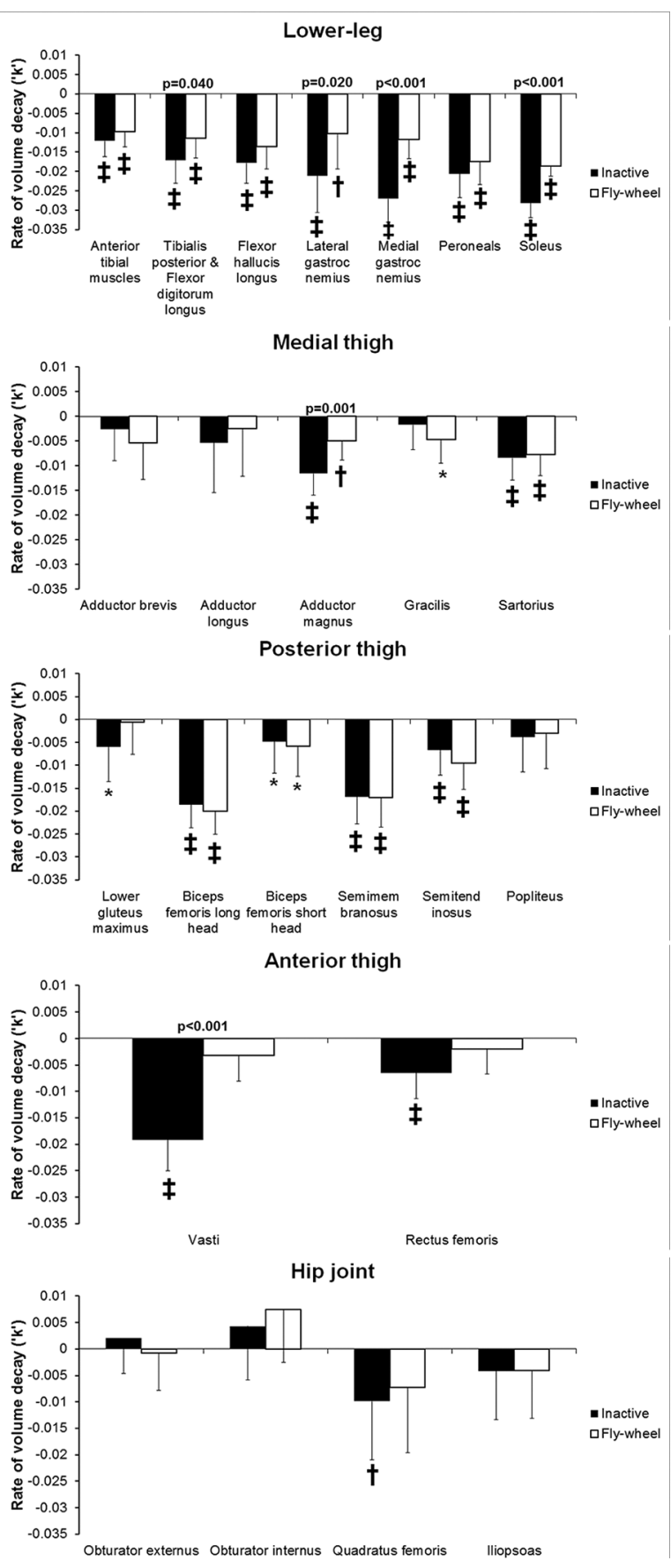

Figure 1 Atrophy and impact of exercise on muscle atrophy values are mean (SD) time constants (' $k$ ') of exponential decay of muscle volume per week of bed-rest. *: $p<0.05$; $\uparrow$ : $p<0.01 ; \ddagger: p<0.001$ and indicate significance of difference of time constant to zero (ie, whether atrophy occurred). $p$ Values above the columns show where the difference between groups was significant. All $p$ values are adjusted for false positives via the 'false discovery rate' method.

group compared with $29 \%$ loss in the inactive group at the end of bed-rest. Prior work does suggest that higher volumes of calf muscle exercise do appear to have a greater protective effect: when resistive vibration exercise including calf press exercises was performed three times a week, ${ }^{19} 15 \%$ loss in soleus muscle volume after 56 days of bed-rest was observed, compared with $7 \%$ loss 
Table 1 Muscle volume changes in the lower leg, anterior thigh

\begin{tabular}{|c|c|c|c|c|c|c|c|}
\hline \multirow[b]{2}{*}{ Group } & \multirow[b]{2}{*}{ Baseline } & \multicolumn{2}{|l|}{ Bed-rest } & \multicolumn{4}{|l|}{ Recovery } \\
\hline & & 28 days $(\%)$ & 89 days $(\%)$ & 13 days $(\%)$ & 90 days $(\%)$ & 180 days $(\%)$ & $360 d(\%)$ \\
\hline \multicolumn{8}{|c|}{ Anterior tibial muscles } \\
\hline Inactive & $252.1(24.9)$ & $-7.0(4.0) \ddagger$ & $-13.6(4.9) \ddagger$ & $-5.1(3.9) \ddagger$ & $-0.3(3.9)$ & $0.1(3.9)$ & $0.4(4.7)$ \\
\hline Flywheel & $246.9(11.8)$ & $-5.1(4.0) \ddagger$ & $-11.2(4.0) \ddagger$ & $-3.0(4.6)$ & $0.5(4.5)$ & $1.2(4.2)$ & $0.4(4.5)$ \\
\hline \multicolumn{8}{|c|}{ Flexor digitorum with tibialis posterior } \\
\hline Inactive & $136.6(25.3)$ & $-11.4(7.4) \ddagger$ & $-19.0(10.4) \ddagger$ & $-7.3(6.7) \ddagger$ & $1.8(5.4)$ & $0.5(4.2)$ & $0.9(4.0)$ \\
\hline Flywheel & $133.5(28.5)$ & $-7.1(6.4) \dagger$ & $-13.0(7.9) \ddagger$ & $-6.0(7.2)^{\star}$ & $-1.2(5.0)$ & $-0.5(4.6)$ & $1.0(5.1)$ \\
\hline \multicolumn{8}{|c|}{ Flexor hallucis longus } \\
\hline Inactive & $80.2(13.4)$ & $-9.5(7.0) \ddagger$ & $-19.6(8.9) \ddagger$ & $-14.7(9.8) \ddagger$ & $0.0(6.5)$ & $1.8(7.5)$ & $2.1(9.0)$ \\
\hline Flywheel & $78.3(8.7)$ & $-8.8(9.8)^{\star}$ & $-15.5(8.4) \ddagger$ & $-10.1(8.4) \ddagger$ & $-4.8(7.1)$ & $-2.9(8.6)$ & $-2.2(9.3)$ \\
\hline \multicolumn{8}{|c|}{ Lateral gastrocnemius } \\
\hline Inactive & $132.6(20.7)$ & $-10.7(10.3) \ddagger$ & $-23.3(13.0) \ddagger$ & $-11.8(11.6) \ddagger$ & $2.6(9.6)$ & $1.1(10.1)$ & $3.0(10.5)$ \\
\hline Flywheel & $140.2(15.2)$ & $-4.0(8.4))$ & $-12.8(10.7) \ddagger$ & $-7.5(9.8)^{\star}$ & $2.3(8.4)$ & $-0.8(7.0)$ & $-1.4(8.2)$ \\
\hline \multicolumn{8}{|c|}{ Medial gastrocnemius } \\
\hline Inactive & $222.1(40.6)$ & $-15.6(7.6) \ddagger$ & $-28.0(12.3) \ddagger$ & $-7.6(8.4) \ddagger$ & $2.7(6.4)$ & $2.5(4.8)$ & $3.9(6.0)^{*}$ \\
\hline Flywheel & $235.2(30.6)$ & $-5.6(5.2) \dagger$ & $-13.3(7.3) \ddagger$ & $-5.1(6.6)^{\star}$ & $1.7(4.0)$ & $0.9(3.2)$ & $0.0(3.0)$ \\
\hline \multicolumn{8}{|l|}{ Peroneals } \\
\hline Inactive & $136.9(29.0)$ & $-12.1(6.4) \ddagger$ & $-22.5(11.8) \ddagger$ & $-12.8(7.7) \ddagger$ & $-0.1(4.6)$ & $0.7(4.2)$ & $1.6(4.9)$ \\
\hline Flywheel & $140.9(29.2)$ & $-10.3(4.7) \ddagger$ & $-18.4(6.3) \ddagger$ & $-9.0(6.0) \ddagger$ & $0.1(6.7)$ & $-2.0(8.1)$ & $-0.5(7.9)$ \\
\hline \multicolumn{8}{|l|}{ Soleus } \\
\hline Inactive & $449.4(61.0)$ & $-15.6(6.1) \ddagger$ & $-28.6(8.5) \ddagger$ & $-5.5(6.1) \ddagger$ & $2.4(4.6)$ & $3.9(3.2) \ddagger$ & $4.3(5.0) \dagger$ \\
\hline Fly-wheel & $496.4(64.5)$ & $-10.6(3.0) \ddagger$ & $-19.9(4.5) \ddagger$ & $-5.5(4.7) \dagger$ & $1.3(3.8)$ & $0.7(4.5)$ & $1.2(3.5)$ \\
\hline \multicolumn{8}{|l|}{ Vasti } \\
\hline Inactive & $1688.8(203.7)$ & $-12.1(6.0) \ddagger$ & $-20.3(7.3) \ddagger$ & $-10.1(6.7) \ddagger$ & $0.9(7.5)$ & $1.5(6.8)$ & $3.1(8.5)$ \\
\hline Flywheel & $1670.2(115.0)$ & $-1.5(4.3)$ & $-3.8(5.8)$ & $-0.5(6.1)$ & $1.6(2.1)$ & $1.0(3.2)$ & $-0.5(2.4)$ \\
\hline \multicolumn{8}{|c|}{ Rectus femoris } \\
\hline Inactive & $259.3(33.0)$ & $-3.6(5.3)^{\star}$ & $-7.9(6.2) \ddagger$ & $-4.2(6.2)^{\star}$ & $-0.8(6.0)$ & $-0.1(5.4)$ & $1.0(6.9)$ \\
\hline Flywheel & 285.2 (37.3) & $-0.2(3.5)$ & $-2.7(3.7)$ & $0.0(3.9)$ & $0.0(3.8)$ & $2.8(10.4)$ & $0.7(5.4)$ \\
\hline
\end{tabular}

At baseline, values are mean (SD) muscle volume in $\mathrm{cm}^{3}$. During and after bed-rest, values are mean (SD) percentage change in muscle volume compared with baseline. *: $p<0.05 ; \dagger: p<0.01 ; \ddagger: p<0.001$ and indicate significance of difference to baseline. All $p$ values are adjusted for false positives via the 'false discovery rate' method.

in soleus muscle volume after 56 days of bed-rest in another study $^{2}$ where resistive vibration exercise was performed 11 times a week. However, for the calf muscles, there is evidence in the literature ${ }^{31}$ that attaining hypertrophy in ambulant exercise studies is more difficult with standard hypertrophy protocols than it is for the knee extensors. This is in line with the notion that muscles that are typically load-bearing in daily life are more difficult to hypertrophy than nonload-bearing muscles. ${ }^{32}$ Investigation of other exercise modes, such as treadmill running or jumping exercises, is needed to improve exercise prescription for preventing disuse atrophy of the calf musculature.
Recovery of muscle volume occurred in all muscles by 90 days after prolonged bed-rest. We observed, however, increase of muscle volume over and beyond the prebed-rest baseline volume in the inactive subjects in the long term after bed-rest. Importantly, this effect was observed in major muscle groups and not just in minor and/or small muscle groups. The time subjects spent lying supine prior to MR-scanning was strictly controlled in this study. Also, this effect was seen only in the inactive subjects, not in the flywheel exercise subjects (figure 2). We can therefore rule out a systematic, confounding, effect particular to the current study. In further support of our interpretation, peripheral 
Table 2 Muscle volume changes in the medial thigh, posterior thigh and hip

\begin{tabular}{|c|c|c|c|c|c|c|c|}
\hline \multirow[b]{2}{*}{ Group } & \multirow[b]{2}{*}{ Baseline } & \multicolumn{2}{|l|}{ Bed-rest } & \multicolumn{4}{|l|}{ Recovery } \\
\hline & & 28d (\%) & 89d (\%) & 13d (\%) & 90d (\%) & 180d (\%) & 360d (\%) \\
\hline \multicolumn{8}{|c|}{ Adductor brevis } \\
\hline Inactive & $164.1(24.1)$ & $-1.0(6.0)$ & $-2.5(7.3)$ & $-0.8(8.5)$ & $5.6(8.9)$ & $6.0(7.1) \dagger$ & $4.9(7.5)^{\star}$ \\
\hline Flywheel & $186.7(20.2)$ & $-8.2(7.6) \dagger$ & $-4.8(6.8)$ & $-0.4(6.0)$ & $-0.6(5.2)$ & $0.5(6.5)$ & $-2.4(7.1)$ \\
\hline \multicolumn{8}{|c|}{ Adductor longus } \\
\hline Inactive & $159.0(32.5)$ & $-0.7(11.3)$ & $-7.1(12.6)^{\star}$ & $-0.9(14.0)$ & $2.9(9.3)$ & $4.5(10.7)$ & $5.8(12.7)$ \\
\hline Flywheel & $175.8(20.7)$ & $-1.4(7.8)$ & $-2.9(9.1)$ & $-0.9(9.7)$ & $3.2(11.1)$ & $-0.6(11.5)$ & $-1.3(9.9)$ \\
\hline \multicolumn{8}{|c|}{ Adductor magnus } \\
\hline Inactive & $585.8(78.2)$ & $-7.8(5.4) \ddagger$ & $-13.0(6.2) \ddagger$ & $-4.7(4.9) \ddagger$ & $1.6(5.6)$ & $1.4(5.2)$ & $3.0(5.6)$ \\
\hline Flywheel & $586.6(62.5)$ & $-3.0(4.1)$ & $-6.1(3.8) \ddagger$ & $-1.2(3.9)$ & $2.2(2.3)^{\star}$ & $1.3(4.3)$ & $-0.2(3.8)$ \\
\hline \multicolumn{8}{|l|}{ Gracilis } \\
\hline Inactive & $101.6(20.3)$ & $0.2(5.4)$ & $-2.6(5.8)$ & $1.0(7.0)$ & $3.5(6.4)$ & $3.3(5.9)$ & $6.2(9.9)$ \\
\hline Flywheel & $115.2(27.1)$ & $-2.5(5.0)$ & $-6.8(8.5)^{\star}$ & $1.6(7.4)$ & $3.9(6.8)$ & $2.5(6.1)$ & $0.2(5.5)$ \\
\hline \multicolumn{8}{|l|}{ Sartorius } \\
\hline Inactive & $162.3(24.2)$ & $-5.9(4.9) \ddagger$ & $-9.4(5.9) \ddagger$ & $-3.8(5.8)^{\star}$ & $2.3(6.5)$ & $3.8(5.3)^{\star}$ & $5.0(6.3)^{\star}$ \\
\hline Flywheel & 172.8 (11.4) & $-4.3(3.3) \ddagger$ & $-9.1(4.9) \ddagger$ & $-1.7(5.4)$ & $0.8(4.0)$ & $4.9(7.0)$ & $0.5(6.7)$ \\
\hline \multicolumn{8}{|c|}{ Biceps femoris long head } \\
\hline Inactive & $190.2(24.0)$ & $-10.5(6.4) \ddagger$ & $-20.0(7.8) \ddagger$ & $-10.5(7.0) \ddagger$ & $2.7(6.9)$ & $1.7(4.9)$ & $2.2(6.0)$ \\
\hline Flywheel & $234.1(31.3)$ & $-11.8(3.8) \ddagger$ & $-21.1(5.2) \ddagger$ & $-11.7(5.9) \ddagger$ & $0.9(6.6)$ & $0.9(7.2)$ & $0.5(7.3)$ \\
\hline \multicolumn{8}{|c|}{ Biceps femoris short head } \\
\hline Inactive & $97.4(25.0)$ & $-2.8(7.5)$ & $-6.3(8.6) \dagger$ & $-0.2(7.1)$ & $4.5(8.5)$ & $3.0(7.8)$ & $3.1(8.4)$ \\
\hline Flywheel & $104.7(14.9)$ & $-4.4(3.1) \ddagger$ & $-6.8(7.6)^{\star}$ & $-0.1(5.9)$ & $-1.1(5.8)$ & $-0.7(3.9)$ & $-1.5(5.1)$ \\
\hline \multicolumn{8}{|c|}{ Semimembranosus } \\
\hline Inactive & $252.6(43.9)$ & $-10.4(4.1) \ddagger$ & $-18.0(6.3) \ddagger$ & $-8.4(6.5) \ddagger$ & $2.4(4.5)$ & $2.7(3.7)^{\star}$ & $4.3(6.0)^{\star}$ \\
\hline Flywheel & $300.5(50.0)$ & $-10.7(6.8) \ddagger$ & $-18.0(9.0) \ddagger$ & $-9.3(7.5) \ddagger$ & $1.2(6.2)$ & $-0.6(6.5)$ & $1.4(4.7)$ \\
\hline \multicolumn{8}{|c|}{ Semitendinosus } \\
\hline Inactive & $182.3(19.3)$ & $-4.1(5.6)^{\star}$ & $-7.3(6.9) \ddagger$ & $-3.6(6.0)^{\star}$ & $2.2(5.8)$ & $2.2(6.4)$ & $2.0(7.6)$ \\
\hline Flywheel & $189.5(11.6)$ & $-6.5(4.5) \ddagger$ & $-10.6(3.2) \ddagger$ & $-7.1(4.0) \ddagger$ & $0.7(6.3)$ & $-1.1(5.4)$ & $-2.7(6.5)$ \\
\hline \multicolumn{8}{|l|}{ Popliteus } \\
\hline Inactive & $21.0(4.3)$ & $-1.6(6.3)$ & $-5.1(10.2)$ & $-0.4(8.2)$ & $2.4(7.4)$ & $-0.7(6.2)$ & $1.8(8.3)$ \\
\hline Flywheel & $22.1(3.7)$ & $-2.6(7.5)$ & $-4.0(7.8)$ & $2.6(6.0)$ & $-3.4(8.5)$ & $0.3(7.5)$ & $-3.6(5.9)$ \\
\hline \multicolumn{8}{|c|}{ Lower gluteus maximus } \\
\hline Inactive & $535.6(96.6)$ & $-4.1(7.4)$ & $-6.9(8.5) \dagger$ & $-4.5(6.4)^{\star}$ & $1.6(7.2)$ & $0.8(8.1)$ & $5.5(10.9)$ \\
\hline Flywheel & $590.4(80.7)$ & $-0.4(6.1)$ & $-0.5(6.6)$ & $-0.3(7.1)$ & $-2.4(6.6)$ & $-0.8(5.5)$ & $-3.5(7.4)$ \\
\hline \multicolumn{8}{|c|}{ Obturator externus } \\
\hline Inactive & $72.6(10.2)$ & $3.5(6.6)$ & $1.9(7.8)$ & $3.5(8.3)$ & $8.6(8.9) \dagger$ & $7.6(8.7) \dagger$ & $6.4(7.8) \dagger$ \\
\hline Flywheel & $73.8(8.2)$ & $-2.8(7.2)$ & $-0.7(9.6)$ & $3.9(5.7)$ & $2.4(8.4)$ & $0.2(3.9)$ & $3.6(8.0)$ \\
\hline \multicolumn{8}{|c|}{ Obturator internus } \\
\hline Inactive & $57.8(8.5)$ & $5.6(7.8)^{\star}$ & $3.6(9.4)$ & $3.3(9.2)$ & $3.1(9.0)$ & $2.2(8.9)$ & $-1.0(9.4)$ \\
\hline
\end{tabular}




\begin{tabular}{|c|c|c|c|c|c|c|c|}
\hline \multirow[b]{2}{*}{ Group } & \multirow[b]{2}{*}{ Baseline } & \multicolumn{2}{|l|}{ Bed-rest } & \multicolumn{4}{|l|}{ Recovery } \\
\hline & & 28d (\%) & 89d (\%) & 13d (\%) & 90d (\%) & 180d (\%) & 360d (\%) \\
\hline Flywheel & $60.4(7.1)$ & $6.1(6.6)^{\star}$ & $9.4(5.0) \ddagger$ & $9.2(6.8) \dagger$ & $3.0(6.2)$ & $0.2(8.6)$ & $0.3(7.8)$ \\
\hline \multicolumn{8}{|c|}{ Quadratus femoris } \\
\hline Inactive & $36.1(9.5)$ & $-8.8(13.3)^{\star}$ & $-13.5(12.2) \ddagger$ & $-7.8(11.5)^{\star}$ & $-4.5(8.2)$ & $3.9(9.5)$ & $4.7(10.5)$ \\
\hline Flywheel & $40.0(6.7)$ & $-7.7(10.0)^{\star}$ & $-9.8(11.0)^{\star}$ & $-1.0(10.3)$ & $2.0(6.4)$ & $3.7(8.0)$ & $-2.8(9.4)$ \\
\hline \multicolumn{8}{|l|}{ Iliopsoas } \\
\hline Inactive & $71.8(13.1)$ & $-1.0(11.3)$ & $-5.9(11.0)$ & $0.5(9.5)$ & $-1.3(9.0)$ & $0.4(10.0)$ & $1.7(9.7)$ \\
\hline Flywheel & $74.0(16.8)$ & $-5.0(9.3)$ & $-4.3(9.8)$ & $-2.1(12.1)$ & $0.8(11.8)$ & $4.6(11.1)$ & $-1.7(10.2)$ \\
\hline
\end{tabular}

At baseline, values are mean (SD) muscle volume in $\mathrm{cm}^{3}$. During and after bed-rest, values are mean (SD) percentage change in muscle volume compared with baseline. *: $p<0.05 ; \dagger: p<0.01 ; \ddagger: p<0.001$ and indicate significance of difference to baseline. All $p$ values are adjusted for false positives via the 'false discovery rate' method.

quantitative CT examinations were performed in the same collective ${ }^{33}$ and these investigations also showed significant increases in lower leg muscle area 90 days and beyond after bed-rest. After another 56 days bedrest study performed at a different facility, ${ }^{34}$ the authors observed a statistical trend towards an overshoot of calf musculature area recovery after bed-rest, again specific to the inactive group, but not the exercise group, of that study. While we cannot be certain that this muscle hypertrophy involved true muscle fibre hypertrophy, we did not see any association between these muscle volume increases in the long term after bed-rest with improvements in countermovement jump performance.

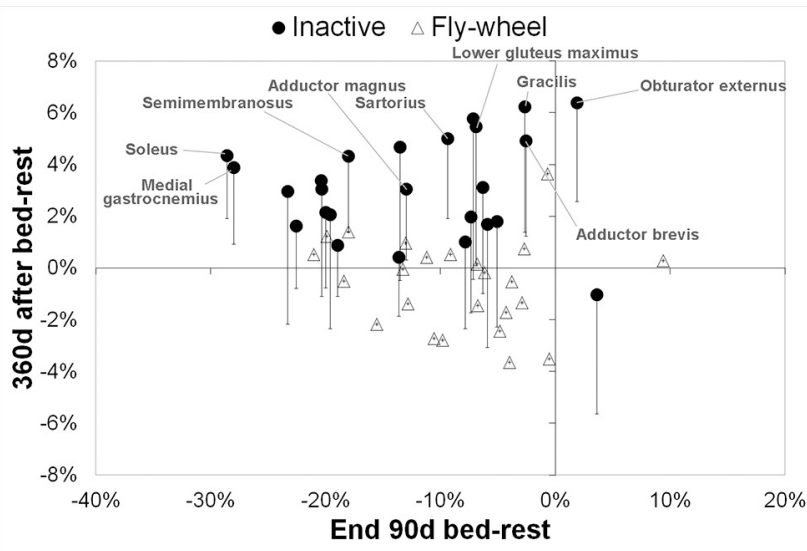

Figure 2 Increases muscle volume 1 year after bed-rest in inactive subjects only: muscle volume change at end bedrest versus muscle volume 1 year after bed-rest. Values are mean percentage difference to baseline at end of bed-rest (x-axis) versus 360 days after bed-rest (y-axis). For the inactive group only, the error bars indicate the unadjusted $95 \% \mathrm{Cl}$ of the mean percentage change 360 days after bedrest. The muscles where the unadjusted $95 \% \mathrm{Cl} 360$ days after bed-rest does not cross zero are labelled. Note that the effect $(A)$ was isolated to the inactive group and (B) did not appear to be related to the extent of muscle loss during bedrest.
Finally, of the muscles active in the hip joint, the quadratus femoris showed the fastest rate and extent, of atrophy. When we observed this in a prior bedrest study, ${ }^{35}$ we were not sure if this was a chance finding. In a recent cadaver study, one group concluded that quadratus femoris is a primary extensor of the hip when it is in a flexed position. ${ }^{36}$ In line with this, recent fine-wire electromyography findings ${ }^{37}$ found that quadratus femoris shows a peak of activity in walking and running during the first part of stance (ie, when the flexed hip begins to bear load) and also in late swing phase in running, presumably to decelerate the flexing hip during late swing. Overall, the recent finding shows that quadratus femoris is important for load-bearing and control of extension force at the hip joint, particularly in a flexed position. This helps to understand why quadratus femoris in particular is affected in disuse.

It is important to mention limitations of the current work. As is typical of bed-rest studies in Europe, only one gender was included in the study in an effort to reduce intersubject variability. As such, we cannot be certain that our results are applicable to female bed-rest participants. The number of subjects was limited due to logistical and financial restraints. Due to the limited number of subjects, some non-significant results for the effect of the exercise protocol may represent false negatives. For some smaller muscles, such as adductor brevis and obturator externus, reproducibility of the measurements is not as high as for larger muscle groups and non-significant findings for these muscles might be false negatives. Even though the current study provides important evidence for exercise prescription in astronauts, application of the current protocols to spaceflight could not occur on a 1:1 basis.

In conclusion, the current study investigated the impact of 90 days bed-rest on atrophy of the muscles of the lower limb, the impact of a flywheel exercise countermeasure, and the recovery of the musculature after 
Table 3 Relationship between muscle atrophy and countermovement jump performance at the end of bedrest and after 180 days of recovery

\begin{tabular}{|c|c|c|}
\hline Muscle & $\begin{array}{l}\text { End of } \\
\text { bed-rest }\end{array}$ & $\begin{array}{l}180 \text { days after } \\
\text { bed-rest }\end{array}$ \\
\hline Anterior tibial muscles & $0.47 a$ & -0.08 \\
\hline $\begin{array}{l}\text { Flexor digitorum with } \\
\text { tibialis posterior }\end{array}$ & $0.53 a$ & -0.38 \\
\hline Flexor hallucis longus & $0.50 a$ & $-0.54 b, *$ \\
\hline Lateral gastrocnemius & $0.64 \mathrm{~b},{ }^{*}$ & -0.01 \\
\hline Medial gastrocnemius & $0.72 \mathrm{c}$, ** & -0.03 \\
\hline Peroneals & 0.22 & -0.16 \\
\hline Soleus & $0.71 \mathrm{c}$, ** & 0.15 \\
\hline Vasti & $0.77 \mathrm{c}$, ,** & -0.09 \\
\hline Rectus femoris & 0.33 & $-0.42 a$ \\
\hline Adductor brevis & -0.04 & -0.17 \\
\hline Adductor longus & 0.28 & -0.38 \\
\hline Adductor magnus & $0.57 \mathrm{~b}, *$ & 0.14 \\
\hline Gracilis & -0.04 & 0.23 \\
\hline Sartorius & 0.00 & -0.38 \\
\hline Lower glut max & -0.05 & 0.23 \\
\hline Biceps femoris long head & 0.03 & -0.13 \\
\hline Biceps femoris short head & -0.22 & -0.39 \\
\hline Semimembranosus & -0.10 & -0.10 \\
\hline Semitendinosus & 0.32 & -0.27 \\
\hline Popliteus & 0.29 & 0.17 \\
\hline Obturator externus & -0.06 & -0.35 \\
\hline Obturator internus & -0.07 & -0.02 \\
\hline Quadratus femoris & 0.26 & -0.27 \\
\hline Iliopsoas & 0.02 & -0.23 \\
\hline
\end{tabular}

Values are Pearson's correlation coefficient. *: $p<0.05 ; * \star p<0.01$; $\star \star \star *: p<0.001$ and indicate significance of correlation with $p$ value adjusted for false positives via the 'false discovery rate' method. a: $p<0.05 ; b: p<0.01 ; c: p<0.001$ indicate significance of correlation with raw $p$ values. Percentage change in muscle volume versus baseline was correlated with percentage change in countermovement jump power versus baseline. A positive (or negative) correlation implies greater (or less) muscle atrophy is associated with greater (or less) loss of countermovement jump power. Strong positive correlations between calf and thigh muscle atrophy were seen at the end of bed-rest, implying a dependence of jump performance loss on atrophy in these muscles. Since no positive correlations were seen 180 days after bed-rest, this implies that increased muscle volume seen in the inactive control group long-term after bed-rest (figure 2; tables 1 and 2) was not associated with functional benefits.

bed-rest. The greatest rates and extent of atrophy was seen in the soleus and gastrocnemius medialis, followed by the gastrocnemius lateralis, peroneals, vasti, biceps femoris long head, other posterior calf musculature, semimembranosus, anterior tibial muscles, adductor magnus and quadratus femoris. The flywheel exercise countermeasure was effective, compared with control, in preventing or reducing atrophy in the vasti, adductor magnus and ankle dorsiflexors/toe flexors. Thus, a short-duration high-intensity resistance exercise programme performed every third day can be effective in preventing muscle atrophy in disuse. The countermeasure was, however, not effective in preventing atrophy of the hamstrings, medial thigh muscles, ankle evertors and dorsiflexors. Finally, in the long term after bed-rest we saw that inactive subjects exhibited an overshoot of muscle volume recovery in some muscle groups compared with before bed-rest.

Acknowledgements The authors thank the subjects for their participation in the study. Centre National d'Etudes Spatiales (CNES) was the 'Promoteur' of the study according to French law and the LTBR study has been performed by MEDES, Institute for Space Physiology and Medicine. The results of the present study do not constitute endorsement by ACSM.

Contributors DLB: analysis of MRI data, statistical analyses, drafting of manuscript. HO: conception and design of the experiments, secured funding, approved final version of the manuscript. JR: conception and design of the experiments, organised data acquisition, secured funding, interpretation of results, revision of manuscript. DF: conception and design of the experiments, secured funding, revision of manuscript.

Funding The participation of Rittweger/Felsenberg in the LTBR study was supported by the German Aerospace Center with grant number 50 WB 0156. The analyses of the image data were supported by grant number 50 WB 1220 from the German Aerospace Center (DLR). The LTBR study was sponsored by the European Space Agency (ESA), the Japan Aerospace Exploration Agency (JAXA) and the Centre National d'Etudes Spatiales (CNES).

\section{Competing interests None declared.}

Ethics approval Toulouse I ethics committee (CCPPRB Toulouse I) of the Rangueil University Hospital as well as the ethical committee of the Free University of Berlin.

Provenance and peer review Not commissioned; externally peer reviewed.

Open Access This is an Open Access article distributed in accordance with the Creative Commons Attribution Non Commercial (CC BY-NC 4.0) license, which permits others to distribute, remix, adapt, build upon this work noncommercially, and license their derivative works on different terms, provided the original work is properly cited and the use is non-commercial. See: http:// creativecommons.org/licenses/by-nc/4.0/

Correction notice This paper has been amended since it was published Online First. Owing to a scripting error, some of the publisher names in the references were replaced with 'BMJ Publishing Group'. This only affected the full text version, not the PDF. We have since corrected these errors and the correct publishers have been inserted into the references.

(c) Article author(s) (or their employer(s) unless otherwise stated in the text of the article) 2017. All rights reserved. No commercial use is permitted unless otherwise expressly granted.

\section{REFERENCES}

1. Nicogossian AE, Dietlein LF. Microgravity Simulation and Analogues. In Nicogossian AE, ed. Space physiology and Medicine. Philadelphia: Lea \& Febiger, 1982:240-8.

2. Belavý DL, Miokovic T, Armbrecht G, et al. Resistive vibration exercise reduces lower limb muscle atrophy during 56-day bed-rest. J Musculoskelet Neuronal Interact 2009:9:225-35.

3. Berg HE, Tesch A. A gravity-independent ergometer to be used for resistance training in space. Aviat Space Environ Med 1994;65:752-6. 
4. Norrbrand L, Pozzo M, Tesch PA. Flywheel resistance training calls for greater eccentric muscle activation than weight training. Eur $J$ Appl Physiol 2010;110:997-1005.

5. Alkner BA, Berg HE, Kozlovskaya I, et al. Effects of strength training using a gravity-independent exercise system, performed during 110 days of simulated space station confinement. Eur J Appl Physiol 2003;90:44-9.

6. Tesch PA, Trieschmann JT, Ekberg A. Hypertrophy of chronically unloaded muscle subjected to resistance exercise. J Appl Physiol 2004;96:1451-8.

7. Irimia JM, Guerrero M, Rodriguez-Miguelez P, et al. Metabolic adaptations in skeletal muscle after 84 days of bed rest with and without concurrent flywheel resistance exercise. J Appl Physiol 2017;122:96-103.

8. Booth FW, Gollnick PD. Effects of disuse on the structure and function of skeletal muscle. Med Sci Sports Exerc 1983;15:415-20.

9. Akima H, Kubo K, Imai M, et al. Inactivity and muscle: effect of resistance training during bed rest on muscle size in the lower limb. Acta Physiol Scand 2001;172:269-78.

10. Akima $\mathrm{H}$, Kubo K, Kanehisa $\mathrm{H}$, et al. Leg-press resistance training during 20 days of 6 degrees head-down-tilt bed rest prevents muscle deconditioning. Eur J Appl Physiol 2000;82:30-8.

11. Akima $\mathrm{H}$, Ushiyama J-ichi, Kubo J, et al. Effect of unloading on muscle volume with and without resistance training. Acta Astronaut 2007;60:728-36.

12. Belavý DL, Miokovic T, Armbrecht G, et al. Differential atrophy of the lower-limb musculature during prolonged bed-rest. Eur J Appl Physiol 2009;107:489-99.

13. Kouzaki M, Masani $\mathrm{K}$, Akima $\mathrm{H}$, et al. Effects of 20 -day bed rest with and without strength training on postural sway during quiet standing. Acta Physiol 2007;189:279-92.

14. LeBlanc AD, Schneider VS, Evans HJ, et al. Regional changes in muscle mass following 17 weeks of bed rest. J Appl Physiol 1992;73:2172-8.

15. Miokovic T, Armbrecht G, Felsenberg D, et al. Heterogeneous atrophy occurs within individual lower limb muscles during 60 days of bed rest. J Appl Physiol 2012;113:1545-59.

16. Shackelford LC, LeBlanc AD, Driscoll TB, et al. Resistance exercise as a countermeasure to disuse-induced bone loss. J Appl Physiol 2004:97:119-29.

17. Zange J, Mester J, Heer M, et al. $20-\mathrm{Hz}$ whole body vibration training fails to counteract the decrease in leg muscle volume caused by 14 days of 6 degrees head down tilt bed rest. Eur J Appl Physiol 2009;105:271-7.

18. Rittweger J, Frost HM, Schiessl $\mathrm{H}$, et al. Muscle atrophy and bone loss after 90 days' bed rest and the effects of flywheel resistive exercise and pamidronate: results from the LTBR study. Bone 2005;36:1019-29.

19. Miokovic T, Armbrecht G, Gast U, et al. Muscle atrophy, pain, and damage in bed rest reduced by resistive (vibration) exercise. Med Sci Sports Exerc 2014;46:1506-16.

20. Rittweger J, Felsenberg D, Maganaris C, et al. Vertical jump performance after 90 days bed rest with and without flywhee resistive exercise, including a 180 days follow-up. Eur J Appl Physio 2007;100:427-36.
21. Matheson LA, Duffy S, Maroof A, et al. Intra- and inter-rater reliability of jumping mechanography muscle function assessments. J Musculoskelet Neuronal Interact 2013;13:480-6.

22. Rittweger J, Schiessl H, Felsenberg D, et al. Reproducibility of the jumping mechanography as a test of mechanical power output in physically competent adult and elderly subjects. J Am Geriatr Soc 2004:52:128-31.

23. Gast $U$, John $S$, Runge $M$, et al. Short-duration resistive exercise sustains neuromuscular function after bed rest. Med Sci Sports Exerc 2012;44:1764-72.

24. Holguin N, Muir J, Rubin C, et al. Short applications of very lowmagnitude vibrations attenuate expansion of the intervertebral disc during extended bed rest. Spine J 2009;9:470-7.

25. Suzuki $\mathrm{Y}$, Kashihara $\mathrm{H}$, Takenaka $\mathrm{K}$, et al. Effects of daily mild supine exercise on physical performance after 20 days bed rest in young persons. Acta Astronaut 1994;33:101-11.

26. Berry $\mathrm{P}$, Berry I, Manelfe $\mathrm{C}$. Magnetic resonance imaging evaluation of lower limb muscles during bed rest-a microgravity simulation model. Aviat Space Environ Med 1993;64:212-8.

27. Akima H, Hotta N, Sato K, et al. Cycle ergometer exercise to counteract muscle atrophy during unilateral lower limb suspension. Aviat Space Environ Med 2009;80:652-6.

28. Ono $T$, Higashihara A, Fukubayashi T. Hamstring functions during hip-extension exercise assessed with electromyography and magnetic resonance imaging. Res Sports Med 2011;19:42-52.

29. Mendiguchia J, Garrues MA, Cronin JB, et al. Nonuniform changes in MRI measurements of the thigh muscles after two hamstring strengthening exercises. J Strength Cond Res 2013;27:574-81.

30. Ono T, Okuwaki T, Fukubayashi T. Differences in activation patterns of knee flexor muscles during concentric and eccentric exercises. Res Sports Med 2010;18:188-98.

31. Ferri A, Scaglioni G, Pousson M, et al. Strength and power changes of the human plantar flexors and knee extensors in response to resistance training in old age. Acta Physiol Scand 2003; 177:69-78.

32. Welle S, Totterman S, Thornton C. Effect of age on muscle hypertrophy induced by resistance training. J Gerontol A Biol Sci Med Sci 1996;51:M270-M275.

33. Rittweger J, Felsenberg D. Recovery of muscle atrophy and bone loss from 90 days bed rest: results from a one-year follow-up. Bone 2009;44:214-24.

34. Rittweger J, Beller G, Armbrecht G, et al. Prevention of bone loss during 56 days of strict bed rest by side-alternating resistive vibration exercise. Bone 2010;46:137-47.

35. Miokovic T, Armbrecht G, Felsenberg D, et al. Differential atrophy of the postero-lateral hip musculature during prolonged bedrest and the influence of exercise countermeasures. J Appl Physiol 2011;110:926-34.

36. Vaarbakken K, Steen H, Samuelsen G, et al. Primary functions of the quadratus femoris and obturator externus muscles indicated from lengths and moment arms measured in mobilized cadavers. Clin Biomech 2015;30:231-7.

37. Semciw Al, Freeman M, Kunstler BE, et al. Quadratus femoris: an EMG investigation during walking and running. J Biomech 2015;48:3433-9. 